\title{
ANISOTROPY OF THE VELOCITY DISPERSION IN $\omega$ CENTAURI
}

\author{
G. Meylan \\ Astronomy Department \\ University of California \\ Berkeley CA 94720 \\ U.S.A.
}

ABSTRACT. By far the brightest and the most massive globular cluster in our Galaxy, $\omega$ Cen seems to be, in some of its properties, a kind of transition step between dwarf ellipticals and ordinary globular clusters. For this giant cluster, the comparison between observations and King-Michie multi-mass dynamical models appears possible only using models with strong anisotropy in the velocity dispersion. A more comprehensive description of this work is to be published (Meylan 1986).

\section{THE OBSERVATIONS}

The present dynamical description of $\omega$ Cen uses both surface brightness and velocity dispersion profiles. Precise radial velocities have been obtained with the photoelectric spectrometer CORAVEL at the European Southern Observatory at Cerro La Silla, Chile, in collaboration with astronomers in Geneva, Marseilles, Copenhagen, and ESO (Mayor et al. 1986). The number of observations amounts to 540 measurements of 318 member stars, with typical uncertainties of $0.9 \mathrm{~km} / \mathrm{s}$.

\section{THE MODEL}

King-Michie dynamical models, based on an assumed form for the phase-space distribution function, have been constructed in an approach nearly identical to that of Gunn and Griffin (1979). In order to mimic a real cluster, heavy remnants (such as stellar black holes or neutron stars), white dwarfs and main sequence stars have been distributed into ten different subpopulations, each having the energy-angular momentum (E,J) distribution function

$$
f_{i}(E, J) \propto\left[\exp \left(-A_{i} E\right)-1\right] \exp \left(-\beta J^{2}\right)
$$

In the cluster center, thermal equilibrium is assumed in order to force $A_{i}$ to be proportional to the mean mass of the stars in the subpopulation considered. A model is specified by a mass function exponent $x$, and by four parameters: the scale radius $r_{c}$, the scale velocity $v_{s}$, the central value of the gravitational potential $W_{\circ}$, and the anisotropy radius $r_{a}$. Beyond $r_{a}$, the velocity dispersion tensor is mostly radial. 


\section{THE RESULTS}

A grid of models has been calculated for a wide range of values of each parameter. The observations are well fitted only by models with strong anisotropy, i.e. with $r_{a} \simeq 2-3 r_{c}$ (Fig. 1 and 2). This is related to the large value of the half-mass relaxation time $t_{r h} \simeq 20-30 \times 10^{9} \mathrm{yr}\left(t_{r}(0) \simeq 1 \times 10^{9} \mathrm{yr}\right)$.

The mean value of the exponent $x$ of the mass function is $\simeq 1.25-1.50$, a value close to Salpeter's value of 1.35 . Heavy remnants represent from 1 to $9 \%$ of the total mass. In models with no remnants at all $\left(m_{h r}=0\right)$, we notice that an increase of the total mass of the white dwarfs is needed to fit the observations. The mean value of the total mass is about $4 \times 10^{6} M_{\odot}$, giving a mean $M / L_{v} \simeq 3$.

\section{REFERENCES}

Gunn, J.E., and Griffin, R.F. 1979, A. J. 84, 752.

Mayor, M., Imbert, M., Anderson, J., Ardeberg, A., Benz, W., Lindgren, H., Martin, N., Maurice, E., Meylan, G., Nordstroem, B., and Prévot, L. 1986 Astron. Astrophys. Suppl. to be published .

Meylan, G. 1986 Astron. Astrophys. in preparation.
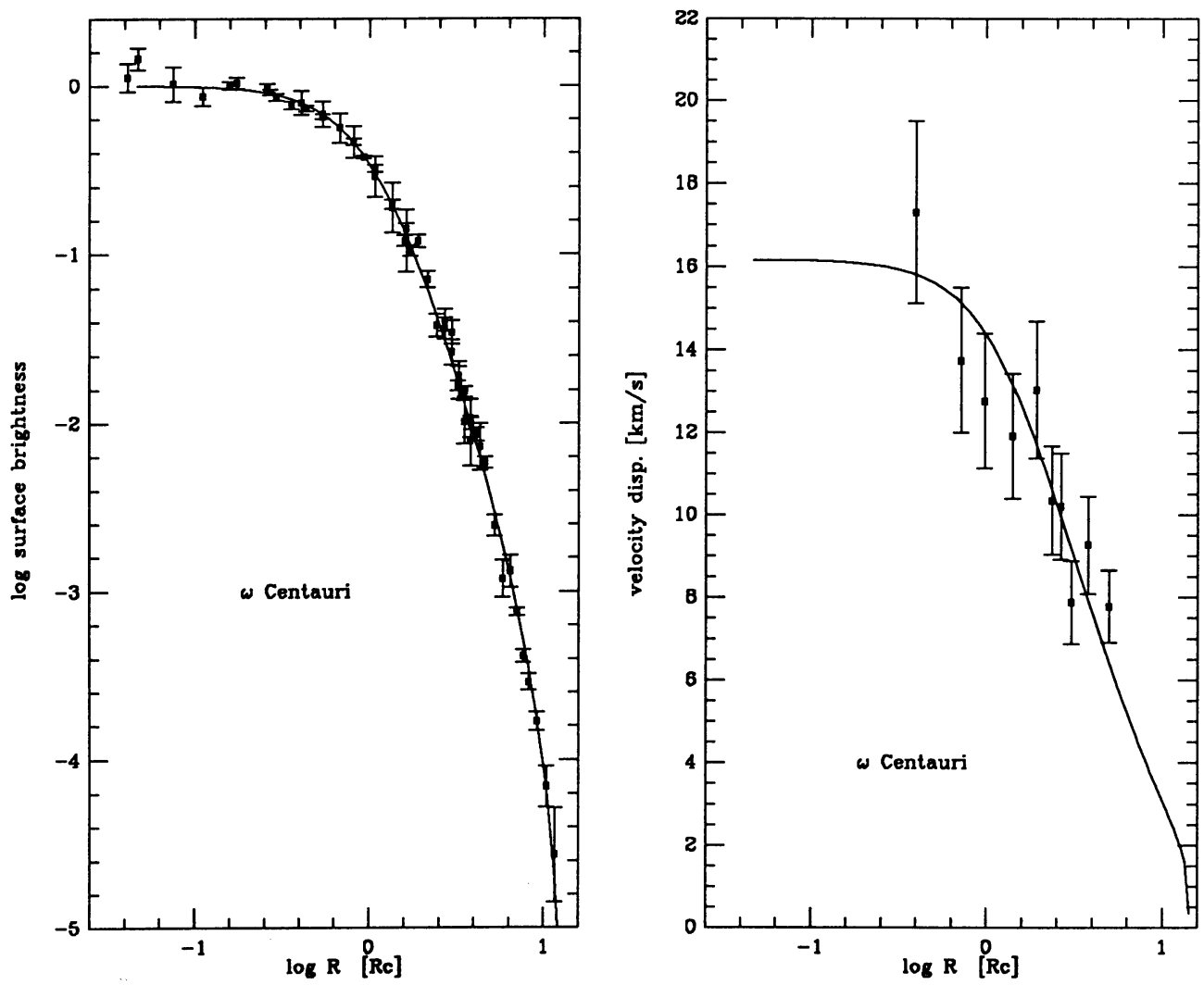

Fig. 1 and 2 Observed and computed surface brightness and velocity dispersion profiles, for the model: $m_{h r}=0 ., \mathrm{x}=1.25, W_{\circ}=5.0$, and $r_{a}=2.0$. 